\title{
Conflictos escolares como factor de riesgo en el rendimiento académico y deserción escolar.
}

Dra. en T. I. E. María de los Ángeles Carmona Zepeda

mamp45297@yahoo.com.mx

Plantel Cuauhtémoc de la UAEM

M. en P. F. S. Leticia Castillón Contreras

letycasti@hotmail.com

Plantel Cuauhtémoc de la UAEM

Dr. en C. S. Rubén Gutiérrez Gómez.

rubengut@yahoo.com.mx

Dirección Media Superior de la UAEM

Recibido: 04 de abril del 2020

Resumen

Aceptado: 15 de mayo del 2020

El conflicto escolar es, como todo conflicto, un factor inherente en las relaciones humanas, por lo tanto es necesario identificar su existencia en el ámbito escolar, con la finalidad de limitar sus repercusiones, las cuales están ligadas al rendimiento académico y, por lo tanto, a la deserción escolar. El presente trabajo realizó una investigación descriptiva en la que participaron 2883 alumnos de tercer y quinto semestre de los diferentes planteles de la Universidad Autónoma del Estado de México que se encuentran localizados en el Valle de Toluca, con el objetivo de detectar los tipos de conflicto y su incidencia como factor de riesgo en la deserción.

Palabras clave: Conflictos escolares, deserción escolar, rendimiento académico.

\section{School conflicts as a risk factor in academic performance and school dropout}

\begin{abstract}
School conflict is, like all conflict, an inherent factor in human relationships, therfore it is necessary to identify its existence in the school life in order to limit its repercussions, which are linked to academic performance and therefore shool dropout. The present work carried out a descriptive investigation in which 2883 third and fifth semester students from the


different establishments of the Autonomous University of the State of Mexico who are located in the Toluca Valley. Our obejtive is detect the type of conflict and its incidence as a risk in desertion factors.

Keywords: School conflicts, school dropout, academic performance.

\section{Introducción:}

El conflicto en las escuelas no es un tema novedoso, pero si es una situación ignorada, debido a que siempre ha estado presente en toda organización humana (Mogan 1990) ya que es inherente a las políticas educativas, currículum, metas sociales y al ser humano.

El conflicto es inevitable en los grupos humanos y los intentos de evadirlos han tenido efectos contrarios, agravándose. Los conflictos escolares no son una excepción. Dichos conflictos poseen un potencial constructivo y destructivo, en dependencia de la manera de enfrentarlos y resolverlos constructivamente. Es verdad que a menudo el conflicto crea tensión, ansiedad y molestia, pero como el enfado, estos sentimientos en sí mismo no son siempre malos.

Pueden proporcionar el tiro y afloja necesario para el desarrollo y el crecimiento. Creemos que el conflicto en el aula es benéfico, ya que presenta una posibilidad de generar soluciones creativas a problemas y motivar a los alumnos a mejorar su rendimiento individual y/o grupal. Incluso podemos decir que es necesario para el aprendizaje personal y el proceso de cambio. (Schmuck y Schmuck, 1983, en Ovejero, 1991). En esta misma dirección afirma Johnson (1978) que el conflicto escolar es necesario para combatir la rutina escolar y facilitar el progreso de enseñanza - aprendizaje.

El conflicto es definido como "lucha, desacuerdo, incompatibilidad aparente, confrontación de intereses, percepciones o actitudes hostiles entre dos o más partes. El conflicto es connatural con la vida misma, está en relación directa con el esfuerzo por vivir. Los conflictos se relacionan con la satisfacción de las necesidades, se encuentra en relación con procesos de estrés y sensaciones de temor y con el desarrollo de la acción que puede llevar o no hacia comportamientos agresivos y violentos (...)” (Vinyamata 2001, p. 129). 
Desde la perspectiva de esta teoría, el conflicto consiste en la incompatibilidad entre los fines que persiguen dos o más actores, que pueden presentar una manifestación violenta o no.

Así mismo, los conflictos contienen tres elementos:

El primer elemento es la actitud. La cual consiste en la construcción, por uno de los actores o por ambos, de una imagen idealizada de uno mismo, unida a una imagen del otro revestida exclusivamente de rasgos negativos (Galtung, 1978 citado en Martínez, 1991).

El segundo aspecto es el comportamiento. La polarización mental, que genera la actitud conflictiva conduce a dos fases de comportamiento conflictivo: la primera, hace referencia a las relaciones personales, y la segunda, el comportamiento conflictivo, es el momento en que un individuo piensa, desea, quiere y actúa para destruir a otra persona (Galtung, 1978 citado en Martínez, 1991).

El tercer elemento, es la incompatibilidad de fines, siendo esta el núcleo central del conflicto. Es decir, el individuo busca obstaculizar todos los planes del otro, por lo tanto los valores de uno obstaculizan los del otro y sus intereses también obstaculizan los intereses de la otra persona. (Galtung 1986 cit. En Martínez, 1991).

La justificación de realizar la investigación es que a pesar de que los conflictos se encuentran presentes en el nivel medio superior en la UAEMéx, son ignorados y se desconoce el impacto que tienen en el rendimiento académico de los alumnos, así como en la deserción escolar.

\section{Marco o fundamentación teórica}

En el presente estudio al hablar de conflictos en el ámbito escolar, con expresión violenta llamados indicadores del conflicto, se hará referencia a la categoría de conflictos escolares propuestas de Torrego y Moreno (2003), haciendo una adaptación de los mismo en el contexto escolar particular de la investigación.

1. Disrupción en las aulas. Son todas las acciones inapropiadas que se realizan en el salón de clases e impiden el desarrollo del proceso de enseñanza - aprendizaje (boicot, ruido permanente, interrupciones, etc.). Estas acciones implican una pérdida de tiempo, se consideran como falta de disciplina y genera el mayor índice de fracaso escolar, además de 
distanciar emocionalmente a profesores y alumnos, dificultando la relación entre ambos (Torrego, 2009).

2. Vandalismo. Son todos los actos de violencia física contra las instalaciones de la escuela (Torrego, 2009).

3. Problemas de disciplina. Es cuando existe una trasgresión a las normas de convivencia dentro y fuera del salón de clases (daños a materiales, incumplimiento de horarios, consumo de tabaco o de otras sustancias, indumentaria inadecuada, y otros aspectos recogidos en los reglamentos de convivencia) (Torrego, 2009).

4. Bullying o acoso escolar. Es un maltrato reiterado o permanente a un estudiante incapaz de defenderse de forma efectiva, generado por otro u otros alumnos. Se genera a través de acciones violentas, ocultas a las autoridades, normalmente practicado en grupo (intimidación directa o indirecta, a través de internet o de los teléfonos móviles, exclusión social, poner motes, vejaciones, esconder cosas, deterioras propiedades de la persona) (Torrego, 2009).

5. Acoso sexual. Se puede considerar una característica de comportamientos antisociales, y es un atentado de la dignidad y libertad sexual de las personas, desafortunadamente, no se conocen muchos datos sobre este factor, o bien esta información no es homogénea (Torrego, 2009).

6. Absentismo y deserción escolar. Es cuando no se realizan las tareas asignadas en tu papel de estudiante o de docente (Torrego, 2009).

7. Fraude - corrupción. Son acciones relacionadas a la trasgresión de comportamientos socialmente reconocidos y aceptados de los estudiantes (copiar, plagio, tráfico de influencias, etc.) (Torrego, 2009).

8. Problemas de seguridad en el centro escolar. Surge cuando el clima escolar se percibe como miedo a sufrir daños. Este miedo puede estar generado por cualquier integrante de la comunidad o fuera de ella, como las bandas conectadas a algún alumno (Torrego, 2009). 


\section{Metodología:}

\section{Objetivo}

Detectar la incidencia de los tipos de conflicto como factor de riesgo de deserción escolar en el nivel medio superior de la Universidad Autónoma del Estado de México.

\section{Planteamiento del problema y pregunta de investigación}

El conflicto es una temática escasamente trabajada en la Institución educativa, por lo cual no se reconoce su existencia, vemos manifestaciones de conflicto con mayor frecuencia, reportados por alumnos y docentes, que ocasiona fricciones constantes, resentimientos y actitudes negativas. Un ambiente hostil perjudica las relaciones que componen la escuela (alumnos- alumnos, docentes-alumnos, directivos-docentes y directivos-alumnos).

Una atmosfera donde el conflicto es visualizado como negativo, en la escuela influye en el ejercicio profesional del equipo docente, asimismo repercute en la percepción que tienen los alumnos sobre el espacio físico de la escuela, la administración escolar, los docentes, sus pares y en el rendimiento académico.

¿Los tipos de conflicto son un factor de riesgo de deserción escolar en el nivel medio superior de la Universidad Autónoma del Estado de México?

¿Con qué frecuencia se presentan los conflictos escolares en el nivel medio superior de la UAEMéx?

\section{Diseño de investigación}

\section{Tipo de estudio}

El tipo de investigación fue no experimental y descriptiva, siendo los estudios descriptivos "pretenden medir o recoger información de manera independiente o conjunta sobre los conceptos o las variables a las que se refieren. Son útiles para mostrar los ángulos o dimensiones de un fenómeno, suceso, comunidad, contexto o situación”. (Hernández 2008, p.102). 


\section{Variables implicadas en el estudio}

Conflicto y deserción escolar

Siendo de tipo discretas y categóricas. Discretas son aquellas, que producen respuestas numéricas de un conteo (Berenson, Levine y Krehbiel, 2001, p, 9) y Categóricas: Estas pertenecen a una clase de medida nominal en donde se categoriza o se asigna un objeto o una subclase o subconjunto de una clase o conjunto (Kerlinger, 1998 p. 41).

\section{Población}

Conformada por 10,000 alumnos inscritos en cinco planteles de la escuela preparatoria del Valle de Toluca de la UAEMéx. En el semestre 2019B, de turnos matutino y vespertino de los semestres $3^{\circ}$ y $5^{\circ}$, con edades de entre 15 y 19 años, sexos masculinos y femeninos, de nivel socioeconómico bajo, medio y medio alto

\section{Muestra}

Conformada por 2883 alumnos de cinco planteles de la escuela preparatoria de la UAEMéx, en el Valle de Toluca de los semestres $3^{\circ}$ y $5^{\circ}$ de ambos turnos con edades de entre 15 y 19 años, de sexo femenino y masculino de nivel socioeconómico bajo, medio y medio alto.

\section{Recogida de información, recogida de datos}

Se llevó a cabo en las siguientes fases:

$1^{\circ}$ Fase Cuantitativa cuestionario para alumnos:

Para la presente investigación se elaboró un cuestionario con 45 ítems, sobre la tipología de conflictos y deserción escolar; donde se midieron los indicadores: disrupción en el aula, vandalismo, ausentismo y deserción, acoso sexual, acoso escolar (bullying), disciplina, fraude-corrupción y problemas de seguridad en el centro; el cual tuvo como finalidad detectar la opinión de los alumnos sobre los indicadores anteriores, derivados de su experiencia en la escuela. 
La elaboración del cuestionario, fue a partir de reactivos de tipo no independiente, que obligan al sustentante a elegir un reactivo u opción que excluya la elección de otros reactivos. Estas formas de escala y reactivos se denominan escalas y reactivos de elección forzada. En escala Tipo Lickert. Los ítems fueron analizados y discriminados utilizado el coeficiente de discriminación del reactivo.

Entre más alto es el índice de discriminación, el reactivo diferenciará mejor a las personas con altas y bajas calificaciones. Si todas las personas de GA contestan correctamente un reactivo entonces $\mathrm{D}=1$ (valor máximo de este indicador); si sucede lo contrario $\mathrm{D}=-1$ (valor máximo negativo); si ambos grupos contestan por igual, $\mathrm{D}=0$ (valor mínimo de discriminación). (Magnuson 2009, p.218) Siendo seleccionados aquellos reactivos que obtuvieron puntajes >0.39 de acuerdo con la recomendación de Ebel y Frisbie (1986) (citados en Backhoff, 2000 p. 14)

Para obtener la fiabilidad del cuestionario, se realizará el cálculo del coeficiente de alfa de Cronbach, el cual requiere de una sola aplicación del instrumento de medición y produce valores que oscilan entre 0 y 1 , donde el coeficiente de 0 significa nula confiablidad y 1 representa un máximo de confiabilidad. Cando más se acerque el coeficiente a cero (0), mayor error habrá en la medición. Su ventaja reside en que no es necesario dividir en dos mitades a los ítems del instrumento de medición (Hernández, Fernández y Baptista, 2006, p. 292).

$2^{\circ}$ Fase Cualitativa entrevista con alumnos:

Con la finalidad de explorar las vivencias de los alumnos se realizaron diversas entrevistas a grupos focales, contando con la participación de 52 alumnos de tercer y quinto semestre.

La metodología de dichas entrevistas consistió en dos etapas, en la primera se les entrego a cada participante un escrito con una definición de las variables y se les preguntó si han viso este fenómeno en la escuela y porque creen que suceda esta situación. En la segunda etapa se invitó a participar a todo los integrantes del grupo focal a discutir sobre la variable comentada. 
Se realizaron grabaciones de audio durante todas las entrevistas, con la finalidad de realizar su posterior transcripción y analizar los datos obtenidos en dichas entrevistas.

\section{Aplicación del instrumento}

La aplicación de los cuestionarios se llevó a cabo en las instalaciones de cada Plantel a través de formulario en Drive. Con los alumnos seleccionados por turno, compartiendo la liga y código QR para su respuesta en medios electrónicos, principalmente a través de teléfono móvil que facilito hacerlo en las aulas.

\section{Plan de análisis de la información/ datos}

Recolección y procesamiento estadístico de los datos en tres fases:

Primera: Después aplicar los cuestionarios, se elaboró la base de datos en el programa estadístico Excel, se obtuvieron porcentajes por indicador.

Segunda: Posterior a la elaboración de la base de datos se realizó una discriminación de reactivos a través de las pruebas estadísticas de coeficiente de discriminación del reactivo y el coeficiente de alfa de Cronbach.

Tercera: Se integró la información de forma descriptiva y se incluyó la información recopilada en las entrevistas con grupos focales.

\section{Integración de los resultados}

Los resultados que arrojó la investigación se integraron de forma descriptiva y específicamente los del cuestionario a través de porcentajes representados, en tablas y por cada indicador de la variable conflictos (Disrupción, vandalismo, acoso sexual, disciplina, acoso escolar, absentismo y deserción escolar, fraude-corrupción y problemas de inseguridad), así como por turno, semestre y sexo. 


\section{Resultados}

Tabla 1. Indicador Vandalismo

\begin{tabular}{c|l|c} 
Indicador & \multicolumn{1}{|c|}{ Conductas } & Frecuencia \\
\hline Vandalismo & $\begin{array}{l}\text { Daños a instalaciones escolares tales como baños, bancas, } \\
\text { sillas, laboratorios, escritorios }\end{array}$ & $94 \%$
\end{tabular}

La tabla 1, Muestra los resultados obtenidos en el indicador Vandalismo, donde la frecuencia de aceptación de conductas que realizan los alumnos del nivel medio superior de la UAEMéx, para dañar instalaciones físicas en sus espacios académicos resulto ser alta con un $94 \%$ Este tipo de conflicto conlleva un acto violento ya que la intención está asociada a dañar deliberadamente las instalaciones puede ser planificado, manifestando el desacuerdo ante el sistema educativo, las autoridades y muestra cierto grado de frustración por parte de los estudiantes.

Estos resultados concuerdan con la información obtenida en las entrevistas, ya que los alumnos refieren que el vandalismo es común en los espacios académicos, manifestándose a través de pintar o rayar los pupitres, las paredes de baños, anuncios, y en ocasiones se dañan los equipos multimedia, se comenta que suele ser un acto que se realiza al sentir aburrimiento durante las clases o cuando desean manifestar una idea.

Tabla II indicador seguridad en el centro escolar

\begin{tabular}{c|l|c}
\multicolumn{1}{c}{ Indicador } & \multicolumn{1}{|c}{ Conductas } & Frecuencia \\
\hline \multirow{4}{*}{$\begin{array}{l}\text { Seguridad en el } \\
\text { centro escolar }\end{array}$} & Seguridad dentro de la escuela & $98.4 \%$ \\
\cline { 2 - 3 } & Peleas fuera de la escuela & $30.2 \%$ \\
\cline { 2 - 3 } & Seguridad alrededor de la escuela & $30 \%$ \\
\cline { 2 - 3 } & Pandillas en la escuela & $26 \%$ \\
\cline { 2 - 3 } & Alumnos con algún tipo de arma & $19.2 \%$ \\
\cline { 2 - 3 } & Robo de objetos personales en la escuela & $86.3 \%$
\end{tabular}

La tabla 2, muestra la frecuencia de opiniones sobre los conflictos relacionados a la seguridad dentro y al redor del centro escolar por parte de los estudiantes de nivel medio superior de la UAEMéx, indicando que el $98 \%$ de los sujetos se sienten seguros dentro de la escuela a pesar de reportar un $86.3 \%$ que se presentan robos de objetos personales, el $26 \%$ manifiesta que existen pandillas y el 19.2\% han observado alumnos con armas, por otra parte indican que se 
sientes inseguros fuera de la escuela un $30 \%$ en los alrededores de la misma, ya que han observado en un $30.2 \%$ peleas fuera.

La información obtenida en los grupos focales concuerda con la tabla anterior, ya que se comentó que en general se sienten seguros dentro de las instalaciones, solo se preocupan cuando traen algo de valor, ya que se los pueden robar, al salir de los planteles no hay tal seguridad, a diferencia de los cuestionarios, aquí se considera que la principal razón por la que no se sienten seguros es porque han sido asaltados, sufren de acoso por los transeúntes y los vendedores ambulantes de los alrededores.

Tabla 3. Indicador Disrupción en el aula

\begin{tabular}{c|l|c} 
Indicador & \multicolumn{1}{|c}{ Conductas } & Frecuencia \\
\hline \multirow{4}{*}{$\begin{array}{c}\text { Disrupción en } \\
\text { las aulas }\end{array}$} & Hacer enojar a docentes de forma deliberada & $47.8 \%$ \\
\cline { 2 - 3 } & Platicar constantemente en clase & $98.2 \%$ \\
\cline { 2 - 3 } & Levantarse sin motivo en clase & $99.1 \%$ \\
\cline { 2 - 3 } & Reírse cuando el profesor explica & $80.2 \%$ \\
\cline { 2 - 3 } & Jugar en clase pin chistes y comentarios sin & $19.2 \%$ \\
\cline { 2 - 3 } & $\begin{array}{l}\text { Interrupción de clase por } \\
\text { sentido }\end{array}$ & $81.3 \%$
\end{tabular}

En la tabla 3 se muestran los resultados del indicador Disrupción en las aulas, mismas que se manifiestan a través de conductas tales como: hacer enojar a los docentes de forma deliberada, que se reporta en un $47.8 \%$, Platicar en clase, siendo la de mayor incidencia con $98.1 \%$, levantarse sin motivo $98.1 \%$, reiré mientras el profesor explica $80.2 \%$ y jugar en clase con $19.2 \%$. Lo anterior implica que los alumnos tienen conductas disruptivas las que tienen como finalidad conocer los límites del docente, llamar la atención de este, interrumpir para ser tomado en cuenta. Sin embargo, no es considerado como acto de violencia a menos que sea recurrente. La disrupción en las aulas constituye la preocupación más directa y la fuente de malestar más importante de los docentes, por las interrupciones constantes de la clase y que entre alumnos que están interesados en la clase también, confundido con la disciplina en el aula.

En los grupos focales comentan, además de las acciones descritas anteriormente, algunas formas de disrupción son llegar tarde a clase e incumplir con el material solicitado, además refieren que es usual que cuando los alumnos interrumpen las clases suelen presentar 
una actitud negativa y/o restadora, por lo que lo consideran que se genera por problemas personales ya que estos alumnos necesitan llamar la atención.

Tabla 4. Indicador Deserción escolar

\begin{tabular}{c|l|c} 
Indicador & \multicolumn{1}{|c|}{ Conductas } & Frecuencia \\
\hline \multirow{3}{*}{$\begin{array}{c}\text { Deserción } \\
\text { escolar }\end{array}$} & Abandonar escuela sin motivo aparente & $47.4 \%$ \\
\cline { 2 - 3 } & Abandonar escuela por problemas con docentes & $28.6 \%$ \\
\cline { 2 - 3 } & Abandono escolar por problemas con compañeros & $25.4 \%$ \\
\cline { 2 - 3 } & He pensado abandonar por problemas con compañeros & $10.8 \%$
\end{tabular}

En la tabla IV se puede observar el indicador Deserción escolar, que muestra el porcentaje de respuesta con algún grado de acuerdo, por parte de los alumnos que respondieron el cuestionario. Este se midió a través de cuatro reactivos. Respecto a si han conocido algún compañero (a) que abandono la escuela son motivo aparente reportan un $47.4 \%$, si algún compañero (a) ha abandonado la escuela por problemas con docentes en $28,6 \%$ abandono por problemas con otros compañeros 25.4 y si han considerado abandonar la escuela por problemas con compañeros en un 10.8\% A pesar de considerarse porcentajes bajos el hecho de que los estudiantes perciban el abandono escolar por problemas con compañeros, docentes y que pudieran hacerlo indica que los conflictos entre los actores institucionales está relacionado con el abandono escolar.

Mientras que en las entrevistas, los alumnos mencionan que las principales causas de la deserción escolar son: el absentismo (generado por malas compañías), embarazos adolescentes y, en menor medida, el acoso escolar.

Tabla 5. Indicador Absentismo escolar

\begin{tabular}{c|l|c} 
Indicador & \multicolumn{1}{|c|}{ Conductas } & Frecuencia \\
\hline \multirow{3}{*}{$\begin{array}{c}\text { Absentismo } \\
\text { escolar }\end{array}$} & $\begin{array}{l}\text { Yo cumplo con tareas, trabajos y actividades escolares } \\
\text { actividades escolares }\end{array}$ & $99.6 \%$ \\
\cline { 2 - 3 } & Los alumnos asisten con puntualidad a clases & $57.6 \%$ \\
\cline { 2 - 3 } & Los compañeros faltan a clases & $54.8 \%$ \\
\hline
\end{tabular}

La tabla 5, muestra los resultados del indicador Absentismo escolar donde los sujetos mostraron respuestas de estar de acuerdo con las conductas relacionadas en el cumplimiento de tareas y trabajos escolares en un $98.6 \%$, sin embargo, opinan que el $57.6 \%$ de sus 
compañeros no lo hacen, que faltan a clases en un $92.2 \%$ y que quienes lo hacen con puntualidad es en un 54:8\% Lo que indica que los alumnos se abstienen en cumplir con sus actividades como estudiante, si bien estas no afectan de forma contundente la convivencia o no resultan ser un grave conflicto de manera personal, pueden estar determinados por la motivación que tiene los estudiantes hacia la escuela y se relacionan con el abandono escolar.

Los alumnos consideraron en los grupos focales que el absentismo es una de las principales causas de la deserción escolar, ya sea por las inasistencias o por no entregar trabajos, estas acciones las atribuyen a la influencia de sus amigos, aunque también se hace énfasis a que hay algunos alumnos que a pesar de que se ausenten de clases o no entreguen trabajos los hacen de forma precavida para no afectar sus calificaciones.

Tabla 6. Indicador Fraude- Corrupción

\begin{tabular}{|c|c|c|}
\hline Indicador & Conductas & Frecuencia \\
\hline \multirow{6}{*}{$\begin{array}{l}\text { Fraude- } \\
\text { Corrupción }\end{array}$} & Copiar en exámenes & $51.8 \%$ \\
\hline & Docentes que soliciten dinero para subir calificación & $13.3 \%$ \\
\hline & Robo de exámenes & $13 \%$ \\
\hline & $\begin{array}{l}\text { Falsificación evidencias de cumplimento trabajo firmas, } \\
\text { sellos }\end{array}$ & $42 \%$ \\
\hline & $\begin{array}{l}\text { Alumnos que ofrecen dinero o regalos para subir } \\
\text { calificación }\end{array}$ & $80 \%$ \\
\hline & Plagios de trabajos y tareas & $81 \%$ \\
\hline
\end{tabular}

En la tabla 6, se observan los resultados obtenidos para el indicador Fraudecorrupción el cual refleja el nivel de acuerdo a la prevalencia en los alumnos de nivel medio superior de la UAEMéx, caracterizado por: la acción de copiar durante los exámenes en un 51:8\%, Robo de exámenes $13.3 \%$, falsificación de firmas de docentes o evidencias de trabajo $42 \%$. El plagio de trabajos y tareas en un $81 \%$ y el soborno de alumnos a maestros $80 \%$, y la más baja con docentes que solicitan dinero o regalos para incrementar calificación en 13.3\%. Esto nos indica que el fraude tiene alta incidencia y esto implica un grado de malestar que provoca conflictos entre compañeros, así como estudiantes- docentes.

Este indicador se considera como una práctica muy común entre los estudiantes de nivel medio, los alumnos comentan que son acciones que les repercuten directamente a ellos, pero justifican estas acciones mencionando que les dejan muchas tareas y trabajo, además 
mencionan que algunos trabajan en contra turno o bien, que los maestros no revisan los trabajo que solicitan.

Tabla 7. Indicador Acoso escolar

\begin{tabular}{c|l|c} 
Indicador & \multicolumn{1}{|c}{ Conductas } & Frecuencia \\
\hline \multirow{4}{*}{ Acoso escolar } & Alumnos que amenazan a otros & $28.2 \%$ \\
\cline { 2 - 3 } & Alumnos que no se defienden al ser molestados & $55.3 \%$ \\
\cline { 2 - 3 } & Agresiones físicas hacia otros estudiantes & $40.4 \%$ \\
\cline { 2 - 3 } & Crear rumores o chismes para aislar & $53.2 \%$ \\
\cline { 2 - 3 } & Poner apodos & $79.7 \%$ \\
\cline { 2 - 3 } & Grupos de estudiantes que intimidan a uno solo & $74.1 \%$
\end{tabular}

En la tabla 7 se ven reflejados los porcentajes de aceptación de las conductas que se relacionan con el acoso escolar en los estudiantes participantes en la investigación. El 28.2\% aceptan que han observado como un grupo de alumnos amenaza a otro u otros estudiantes, sin razón aparente, en $55.3 \%$ los alumnos que son molestados por otros, Un $40.4 \%$ ha sido testigo de agresiones físicas de un estudiante o grupo de estudiantes hacia otro estudiante., aceptan que se crean rumores con la finalidad de aislar a otro estudiante en un $53.2 \%$, respecto a colocar apodos la incidencia se presenta en un 79.7/\% y aquellos alumnos que son molestados o intimidados por un grupo es del 74.1\%. Este conglomerado de conductas se presenta de forma alta, mismas que ocasionan conflictos que implican violencia y que se relacionan en gran medida con la deserción escolar o con las intenciones de abandonar la escuela.

En las entrevistas los alumnos mencionan que el acoso escolar se suele enmascarar de bromas, por lo que le restan importancias, sin embargo, se comenta que si han llegado a conocer a personas que están pensando en cambiar de escuela por esta razón.

Tabla 8. Indicador Acoso y Hostigamiento escolar

\begin{tabular}{c|l|c}
\multirow{2}{*}{ Indicador } & \multicolumn{1}{|c}{ Conductas } & Frecuencia \\
\hline \multirow{4}{*}{$\begin{array}{c}\text { Acoso y } \\
\text { hostigamiento } \\
\text { sexual }\end{array}$} & Compañeros que hacen insinuaciones sexuales & $51.9 \%$ \\
\cline { 2 - 3 } & Hablar en doble sentido & $61.2 \%$ \\
\cline { 2 - 3 } & Tocamientos y caricias inadecuados & $37.5 \%$ \\
\cline { 2 - 3 } & Docentes que hacen insinuaciones sexuales & $21.4 \%$ \\
\cline { 2 - 3 } & Algún docente me ha hecho insinuaciones sexuales & $9.1 \%$ \\
\cline { 2 - 3 } & Docentes que toquen, acarician con fines sexuales & $13.9 \%$
\end{tabular}


La tabla 8 refleja el indicador Hostigamiento escolar, que se caracteriza por presentarse entre iguales en este caso estudiante- estudiante del nivel medio superior, mismo que se manifiesta a través de la aceptación de que compañeros (as) hacen insinuaciones sexuales en un 51:9\%, hablan en doble sentido con fines sexuales en 61.2\%, se presentan tocamientos y caricias que consideran inadecuadas por la intención o la parte del cuerpo en 37.5\%. Respecto al Acoso sexual que se presenta de adulto o persona con poder a alguien inferior en este caso docentes- alumnos, los resultados indican que el $21.4 \%$ de los encuestados considera que hay docentes en sus escuelas que hacen insinuaciones sexuales a los alumnos (as), el 13.9\% manifiestan que hay docentes que hacen caricias a estudiantes con fines sexuales y un $9.1 \%$ está de acuerdo con que algún docente le ha hecho insinuaciones sexuales. Estos porcentajes indican la alta incidencia de acoso y hostigamiento sexual que no expresa de forma abierta ya que se considera inadecuado hablar al respecto y existe el temor de no ser escuchado o estigmatizado, siendo un conflicto constante dentro del ámbito escolar.

Al abordar estos puntos se pudo identificar que los estudiantes no quisieron revelar todo lo que han visto, escuchado o vivido, a pesar de esto se mencionó que si existe el acoso sexual, principalmente por parte de los maestros, y se da a través de miradas, comentarios, albures e incluso frotar o masajear su espalada, a lo que algunos alumnos responden con incomodidad y miedo, pero otros comentan que "por pasar la materia harían lo que fuera"; además existen rumores de que alumnas que han salido con maestros. Mientras que el caso del hostigamiento sexual se ve enmascarado de bromas, pero en ocasiones si es muy evidente que las hostigue un compañero y prefieren cambiar de salón, muy pocas veces lo confrontan.

Tabla 9. Indicador Disciplina

\begin{tabular}{c|l|c}
\multicolumn{1}{c|}{ Conductas } & Frecuencia \\
\hline \multirow{5}{*}{ Indicador } & \multicolumn{1}{|c}{} & $68.4 \%$ \\
\cline { 2 - 3 } & Se matan clases & $71.1 \%$ \\
\cline { 2 - 3 } & Asistencia de alumnos en estado de ebriedad & $70 \%$ \\
\cline { 2 - 3 } & Se abandona la escuela en horarios de clase & $79.9 \%$ \\
\cline { 2 - 3 } & Se juega fuera de los salones en horario de clase & $47.6 \%$ \\
\cline { 2 - 3 } & Agresiones físicas o verbales a docentes & $94.6 \%$
\end{tabular}

La tabla 9 señala los resultados obtenidos para el indicador de conflicto disciplina la cual se caracteriza por conductas aceptadas tales como: matar clases (no asistir a clases todo el 
grupo) en un 68:4\%, asistencia de alumnos en estado de ebriedad 71.1\%, abandonar la escuela durante las clases $70 \%$, jugar en salones en horarios de clase $79.8 \%$, agresiones a docentes ya sean verbales o físicas $47.6 \%$, hablar con groserías o pablaras altisonantes en la escuela con otros compañeros 94.6\%. Estas conductas representan el romper las reglas establecidas en reglamentos escolares e institucionales, mismas que alteran la convivencia y generan conflictos ya sea con los compañeros o con los docentes. Ya que son faltas de respeto incluso considerados graves y con cierto grado de violencia.

Además de la información obtenida en los cuestionarios, los grupos focales agregan que dentro de las faltas más comunes a al reglamento de la institución son: consumir alcohol o drogas dentro de las instalaciones del plantel (sobre todo en los baños), peleas, vender drogas o alimento que contengan droga (galletas, brownie), portar algún arma en las instalaciones (sobre todo navajas), y el robo de objetos y dinero.

\section{Discusión y conclusiones}

Los resultados obtenidos en la investigación muestran que: El conflicto se encuentra presente, en forma frecuente y cotidiana, en la dinámica escolar, en el nivel medio superior, de la escuela preparatoria de la UAEMéx., mismo que se ve reflejado a través de la descripción de los ocho tipos de conflicto explorados.

En base a que el conflicto se presenta de forma natural en el ser humano, como resultado de las interacciones y la discrepancia de intereses, valores o fines que se persiguen, el hecho de que estén presentes los conflictos en el nivel medio superior no es sorprendente más bien, es de tomarse en consideración la frecuencia con que se dan la dinámica que se establece en torno a ellos y las expresiones de violencia que llegan a generarse.

De acuerdo con Galtung los conflictos contienen elementos actitudinales, que se pueden detectar claramente en los sujetos participantes en la muestra, los cuales se manifiestan a través de las expresiones idealizadas de los docentes o alumnos de ellos mismos y su desempeño dentro del contexto educativo, reflejando sentimientos y creencias, negativas del otro; que los llevan a manifestar comportamientos que obstaculicen al otro, incompatibilidad de valores que genera un conflicto entre actores consientes y que planifican 
una estrategia, así como la incompatibilidad de intereses que incide en el conflicto estructural, siendo en este caso los actos de obstaculizar las clases e impedir su desarrollo, destruir mobiliarios e instalaciones escolares, incumplir con las normas disciplinarias, no asistir a clases, incumplir con tareas y trabajos, hacer uso de la sexualidad como forma de control, molestar a compañeros, cometer actos fraudulentos, asociarse para cometer ilícitos, insultos, amenazas, etc. En este sentido Ortega (2000) señala que: muchos de los conflictos interpersonales entre docentes y estudiantes tienen su origen en mal entendidos relacionados con lo académico, interpretadas erróneamente, cuando no directamente en el desprecio de unos a los otros dados sus respectivos roles en el proceso instructivo.

"Lo cierto es que la disrupción en las aulas es probablemente el fenómeno, entre todos los estudiados, que más preocupa al profesorado en el día a día de su labor, y el que más gravemente interfiere con el aprendizaje de la gran mayoría de los alumnos de nuestros centros" (Olmedilla 1998).

Desde esta perspectiva teórica también se podría explicar el acoso escolar donde los alumnos han aprendido patrones de víctima, acosador u observador, que tendrán repercusiones en su actuar posterior donde la víctima aprende que no hay escapatoria y permite las humillaciones y maltratos, el acosador manifiesta lo aprendido a través de ejercer poder sobre el más débil y el observador puede incluso reproducir el modelo del abusador en situaciones donde considere que no habrá consecuencias negativas, perpetuando el circulo de maltrato. Por lo que "El maltrato suele desorganizar a la persona y perturbar seriamente sus relaciones con lo que lo rodea. Las repercusiones negativas del bullying se extienden también a los agresores, cuyos sentimientos pueden pasar de la satisfacción inicial a la culpabilidad. Por otro lado, se produce un refuerzo de la violencia en los «verdugos», que se puede generalizar con facilidad a otras situaciones. En el caso de los espectadores, no es extraño que haya una pérdida de sensibilidad ante el sufrimiento ajeno, o una vivencia de desvalimiento semejante a la del compañero maltratado. (Martínez-Otero 2005 p 14). En este mismo se sentido se podría argumentar el aprendizaje de las situaciones de inseguridad en el centro escolar, los alumnos han discriminado que son víctimas potenciales de las pandillas y de ser robadas o sus pertenecías ya sea dentro o fuera de la escuela, respondiendo con 
actitudes de sumisión ante el poder que ejercen ya sea física o psicológicamente sus agresores.

Asimismo se ubica el aprendizaje de conflictos relacionados con el fraudecorrupción, si los alumnos observan a otros compañeros, falsificar firmas, sobornar a docentes, copiar en exámenes, etc., reproducirán las mismas conductas, ya que la experiencia se ubica en que las consecuencias son positivas y se reportan en obtener mejores calificaciones y beneficios académicos ya que la reglamentación no se aplica, lo que seguirán reproduciendo y generalizando otros ámbitos de si vida.

En cuanto al vandalismo, tiene una explicación donde la frustración de los alumnos, ante situaciones académicas y de inconformidad por su papel dentro de la escuela asociada a la falta de identidad hacia la misma, lo lleva a un estado de ira, que se manifiesta a través de la cólera y la exteriorizan por medio del daño a las instalaciones escolares, ya que se presentan sobre todo en época de evaluaciones. "Una visita a algunos centros escolares nos permitiría ver el alcance del espíritu de destrucción que guía a algunos alumnos: mesas, cristales, paredes y armarios destrozados; grafitos obscenos, amenazantes o insultantes; quema de libros, etc.)". (Martínez-Otero p, 15).

Los tipos de conflicto asociados a la disciplina, como al absentismo y deserción escolar, donde el microsistema de los alumnos y docentes caracterizados por su historia de vida, familia, creencias personales, se relacionan con el exosistema escolar, donde las normas y políticas escolares, son percibidas como violentas y ocasionan una reacción similar. “A veces se producen desórdenes de la vida en las aulas, que constituyen un serio problema escolar: incumplimiento de tareas, retrasos injustificados, falta de reconocimiento de la autoridad del profesor, etc., en ocasiones, incluso, se llega al desafío, a la amenaza y a la agresión del alumno hacia el profesor, o de este hacia aquel”' (Ortega 1996, p.246) El hecho de que los alumnos se tengan que someter a normas establecidas de antemano donde, no son considerados o que se establezcan prácticas de enseñanza que no les llaman la atención y contenidos de asignaturas poco articuladas con su realidad, derivan en conductas como la antes mencionadas. Se trata por tanto de una forma de ejercicio de poder, que, al no ser 
democrático, ocasiona una falta de conocimiento de la identidad escolar, si lo que está bien o mal puede o no hacerse no ha sido discutido es difícil asumirlo como propio.

El acoso sexual, donde los alumnos del sexo masculino, lo utilizan al hablar en doble sentido, hacer insinuaciones sexuales, tocarse los genitales, etc., como una forma de fortaleza, superioridad y dominación ante el mismo sexo y de atracción sexual hacia el sexo opuesto. Sin embargo, en este sentido el aprendizaje social retoma matices de aprendizaje por observación de conductas consideradas, como masculinas, que deben cubrir con el rol social esperado y deben sr reproducidas si se quiere tener éxito y aceptación. Ahora bien, el acoso sexual por parte de los docentes hacia las alumnos, se puede ubicar también en el aprendizaje social pero adquiere una connotación de poder, se trata de un fenómeno de manifestación oculta, del cual existen pocos datos e investigaciones reportadas debido a la naturaleza del mismo.

\section{Referencias bibliográficas}

Berenson L. M; Levine D. M.; Krehbiel C.T. (2001): "Estadística para la administración”. México. Pearson

Galtung, J. (1989): "Solving Conflicts: A Peace Research Perspective”. Honolulu, University of Hawaii Press.

Hernández Sampieri, R.; Fernández Collado, C.; Baptista Lucio, Pilar (2003): “Metodología de la investigación”. México, Mc Graw Hill

Johnson D. (1978): "Psicología social de la educación”. Buenos Aires, Kapeluz

Kerlinger N. F. (2002): “Investigación del comportamiento”. México, Mc Graw Hill

Magnuson, D. (2009): “Teoría de los test”. México, Trillas

Martínez-Otero P., V. (2005): “Conflictividad escolar y fomento de la convivencia en revista Iberoamericana de educación”, 38, pp. 12-21

Martínez-Otero P., V. (1991: "Los adolescentes ante el estudio. Causas y consecuencias del rendimiento académico". España, Fundamentos 
Olemdilla Moreno, J.M. (1998): “Comportamiento antisocial en los centros escolares: una visión desde Europa”, en revista Iberoamericana de Educación. 18 pp. 85- 103

Ovejero Bernal, A. (1989): "Psicología social de la educación”. Barcelona, Editorial Herder

Torrego J.C. (Coord); Aguado J.C.; Arribas J.M.; Escaño J.; Fernández I.; Funes S.; Gil M.; Palmeiro C.; Romero G.; De Vicente J.; Villaoslada E. (2009): “Modelo integrado de mejora de la convivencia. Estrategias de mediación y tratamiento de conflictos". España, Graó

Ortega, H. (1996) La teoría y la resolución de conflictos. Programa Cultural de la Paz la democracia en América Central. Costa Rica. Universidad para la Paz. Textos Básicos \#8.

Vinyamata, Camp E. (2003): “Reseña de aprender del conflicto, conflictología y educación” en revista Convergencia. 10, pp. 315-328 